\title{
Modelling to Engineering Data: Using a New Two-Parameter Lifetime Model
}

\author{
Mashail M. AL Sobhi (iD \\ Department of Mathematics, Umm-Al-Qura University, Makkah 24227, Saudi Arabia \\ Correspondence should be addressed to Mashail M. AL Sobhi; mmsobhi@uqu.edu.sa \\ Received 30 December 2021; Revised 1 February 2022; Accepted 4 February 2022; Published 8 March 2022 \\ Academic Editor: Hüseyin Işık
}

Copyright (c) 2022 Mashail M. AL Sobhi. This is an open access article distributed under the Creative Commons Attribution License, which permits unrestricted use, distribution, and reproduction in any medium, provided the original work is properly cited.

\begin{abstract}
A novel two-parameter continuous lifespan model is developed, based on a truncated Fréchet produced family of distributions known as the truncated Fréchet inverted Lindley distribution. It includes a thorough discussion of statistical features such as the quantile function, moments, order statistics, incomplete moments, and Lorenz and Bonferroni curves. The greatest likelihood approach for estimating population parameters is described. Finally, one real-world data set to application is utilized to demonstrate the new distribution's utility. The data represent the tensile strength, measured in GPa, of 69 carbon fibers tested under tension at gauge lengths of $20 \mathrm{~mm}$.
\end{abstract}

\section{Introduction}

Adding parameter(s) to baseline distributions is a traditional approach for generating families of probability distributions. These families have the capacity to increase the desirable aspects of probability distributions as well as extract additional information from a variety of data sets, which may be used in a variety of fields such as engineering, economics, biology, and environmental sciences. Another generator utilizes the shortened random variable. In this context, significant research on the truncated (T)-G families is the T Fréchet-G [1], T Weibull-G [2], Type II T Fréchet-G (TIITFG) [3],T Burr X-G [4], T Lomax-G [5], T power Lo-G (TPLoG) [6], TX family of distributions [7], T log-logistic-G [8], generalized odd Weibull-G [9], Topp-Leone-G [10], transmuted odd Fréchet-G [11] and truncated Cauchy power [12].

Aldahlan [3] proposed the TIITFG family with the following cumulative distribution function (cdf):

$$
F(z ; b, \varphi)=1-e e^{-(1-G(z ; \varphi))^{-b}},
$$

and the density function (pdf)

$$
f(z ; b, \varphi)=\operatorname{beg}(z ; \varphi)(1-G(z ; \varphi))^{-b-1} e^{-(1-G(z ; \varphi))^{-b}} .
$$

The following exponential series is used to generate the expansion of pdf (2):

$$
e^{-c z}=\sum_{j=0}^{\infty} \frac{(-1)^{j} c^{j}}{j !} z^{j}, \quad c>0 .
$$

Regarding the existing binomial series can be used,

$$
(1-Z)^{-c}=\sum_{i=0}^{\infty}\left(\begin{array}{c}
c+i-1 \\
i
\end{array}\right) Z^{i}, \quad c>0 \text {, and }|Z|<1 .
$$

Employing (3) and (4) in (2), then the pdf of TIITFG, where $b$ is real, is

$$
f(z ; b, \varphi)=\sum_{i=0}^{\infty} \eta_{i} g(z ; \varphi) G(z ; \varphi)^{i+1},
$$

where

$$
\eta_{i}=\sum_{j=0}^{\infty} \frac{(-1)^{j}}{j !} b e(-1)^{i}\left(\begin{array}{c}
b(j+1)+i \\
i
\end{array}\right)
$$

The quantile function $Q_{(u)}$ of $X$ is given by 


$$
Q_{(u)}=G^{-1}\left\{1-\left[\ln \left(\frac{e}{1-u}\right)\right]^{(-1 / b)}\right\} .
$$

Sharma et al.[13] investigated the inverted Lindley (ILi) distribution, which has the following pdf and cdf, respectively

$$
\begin{gathered}
g(z ; \theta)=\frac{\theta^{2}}{1+\theta}\left(\frac{1+z}{z^{3}}\right) e^{(-\theta / z)}, \quad z>0, \theta>0, \\
G(z ; \theta)=\left(1+\frac{\theta}{(1+\theta) z}\right) e^{(-\theta / z)}, \quad z>0, \theta>0 .
\end{gathered}
$$

Many statisticians have generalized the ILi distribution in recent years, such as [14] who studied the extended ILi distribution, [15] who proposed the generalized ILi distribution, [16] who proposed the power ILi distribution, a new extension of Ili proposed by $[17,18]$ who studied weighted Ili distribution, [19] who studied alpha power transformed ILi (APTILi) distribution, and [20] who studied extended exponentiated ILi distribution, and logarithmic ILi model was studied by [21] and generalized Marshall Olkin ILi by [22].

The main goal of this article is to present the Type II truncated Fréchet inverted Lindley (TIIFILi) distribution, a novel two-parameter life-time model. The new model is very flexible. The pdf can be symmetric, right skewness, and unimodal. Also, the hrf can be unimodal, increasing, and J-shaped. Investigate some of its statistical features to discuss the statistical inference of the TIIFILi model and to give leading fits than some known models with favourable results for the TIIFILi model.

The new model is extremely adaptable, and we may obtain the cdf and pdf by adding (8) and (9), as shown in (1) and (2).

$$
\begin{aligned}
& F(z ; b, \theta)=1-e e^{-\left(1-(1+(\theta /(1+\theta) z)) e^{(-\theta / z)}\right)^{-b}}, \quad z>0, b, \theta>0 \\
& f(z ; b, \theta)=\frac{\theta^{2} b e}{1+\theta}\left(\frac{1+z}{z^{3}}\right) e^{(-\theta / z)}\left(1-\left(1+\frac{\theta}{(1+\theta) z}\right) e^{(-\theta / z)}\right)^{-b-1} e^{-\left(1-(1+(\theta /(1+\theta) z)) e^{(-\theta / z)}\right)^{-b}} .
\end{aligned}
$$

Using the three equations (3)-(5), we can rewrite (11) as follows:

$f(z ; b, \theta)=\sum_{i=0}^{\infty} \eta_{i} \frac{\theta^{2}}{1+\theta}\left(\frac{1+z}{z^{3}}\right) e^{(-\theta(j+1) / z)}\left(1+\frac{\theta}{(1+\theta) z}\right)^{j}$.

We may rewrite the above equation using the binomial expansion as

$$
f(z ; b, \theta)=\sum_{k=0}^{\infty} \mathbb{C}_{k}\left(\frac{1+z}{z^{k+3}}\right) e^{(-\theta(j+1) / z)}
$$

where

$$
\mathbb{C}_{k}=\sum_{i=0}^{\infty} \eta_{i}\left(\begin{array}{l}
j \\
k
\end{array}\right) \frac{\theta^{k+2}}{(1+\theta)^{k+1}}
$$

The TIIFILi distribution function, the hazard rate function (hrf), the inverted hazard rate function, and the cumulative hazard rating function are given when a random variable $\mathrm{Z}$ follows the TIIFILi model,

$$
\begin{aligned}
& R(z ; b, \theta)=e e^{-\left(1-\left(1+\frac{\theta}{(1+\theta) z}\right) e^{(-\theta / z)}\right)^{-b}}, \\
& h(z ; b, \theta)=\frac{\theta^{2} b}{1+\theta}\left(\frac{1+z}{z^{3}}\right) e^{(-\theta / z)}\left(1-\left(1+\frac{\theta}{(1+\theta) z}\right) e^{(-\theta / z)}\right)^{-b-1}, \\
& \tau(z ; b, \theta)=\frac{\left(\theta^{2} b e / 1+\theta\right)\left((1+z) / z^{3}\right) e^{(-\theta / z)}\left(1-(1+(\theta /(1+\theta) z)) e^{(-\theta / z)}\right)^{-b-1} e^{-\left(1-(1+(\theta /(1+\theta) z)) e^{(-\theta / z)}\right)^{-b}}}{1-e e^{-\left(1-(1+(\theta /(1+\theta) z)) e^{(-\theta / z)}\right)^{-b}}}
\end{aligned}
$$


and

$$
H(z ; b, \theta)=\frac{1}{1-\left(1-(1+(\theta /(1+\theta) z)) e^{(-\theta / z)}\right)^{-b}} .
$$

Figures 1 and 2 show the pdf and hrf plots of the TIIFILi distribution.

Figure 1 demonstrates how the pdf might be unimodal and tilted to the right. Figure 2 depicts various potential hrf forms, including monotone increasing, up-side-down, and J-shaped.

The remainder of this article is arranged as follows: Section 2 investigates distribution's mathematical characteristics of the proposed model. Section 3 covers the estimate of distribution parameters using the maximum likelihood method of estimation. Section 4 provides actual data applications to illustrate the potential of the new distribution, and Section 5 ends with remarks.

\section{Mathematical Properties}

In this section, we will study some statistical properties such as the quantile function, median, order statistics, moments, moment generating function, incomplete moments, and Lorenz and Bonferroni curves.

The quantile function

$$
\left(1+\frac{\theta}{(1+\theta) Q(u)}\right) e^{(-\theta / Q(u))}=1-\left[\ln \left(\frac{e}{1-u}\right)\right]^{(-1 / b)}, \quad 0<u<1 .
$$

By multiplying (9) both sides by $-(1+\theta) e^{-(1+\theta)}$,

$$
-\left(1+\theta+\frac{\theta}{Q(u)}\right) e^{-(1+\theta+(-\theta / Q(u)))}=-(1+\theta) e^{-(1+\theta)}\left(1-\left[\ln \left(\frac{e}{1-u}\right)\right]^{(-1 / b)}\right) .
$$

Then,

$$
Q(u)=-\left[1+\frac{1}{\theta}+\frac{1}{\theta} W_{-1}\left(-(1+\theta) e^{-(1+\theta)}\left(1-\left[\ln \left(\frac{e}{1-u}\right)\right]^{(-1 / b)}\right)\right)\right]^{-1}
$$

Corollary 1. If $Z \sim$ TIITFILi, the median $M$ of $Z$ is given by

$Q(u)=-\left[1+\frac{1}{\theta}+\frac{1}{\theta} W_{-1}\left(-(1+\theta) e^{-(1+\theta)}\left(1-[\ln (2 e)]^{(-1 / b)}\right)\right)\right]^{-1}$.

Assuming $Z_{1}<Z_{2}<\cdots<Z_{n}$ is an order sample from TIITFILi population, the pdf of the $i^{\text {th }}$ ordered statistics is given as

$$
f\left(z_{i: n}\right)=\frac{n !}{(i-1) !(n-i) !} f(z ; b, \theta) F(z ; b, \theta)^{i-1}(1-F(z ; b, \theta))^{n-i} .
$$

Substituting (10) and (11), and applying general binomial series expansion, (13) becomes

$$
\begin{array}{r}
f\left(z_{i: n}\right)=\frac{n ! \theta^{2} b e^{n-i+1} e^{(-\theta / z)}}{(i-1) !(n-i) !(1+\theta)}\left(\frac{1+z}{z^{3}}\right)\left(1-\left(1+\frac{\theta}{(1+\theta) z}\right) e^{(-\theta / z)}\right)^{-b-1} e^{-(n-i+1)\left(1-(1+(\theta /(1+\theta) z)) e^{(-\theta / z)}\right)^{-b}} \\
\left(1-e e^{-\left(1-(1+(\theta /(1+\theta) z)) e^{(-\theta / z)}\right)^{-b}}\right)^{i-1} .
\end{array}
$$

We can get the first and last order statistics at $i=1$ and $i=n$, respectively, as follows:

$$
f\left(z_{1: n}\right)=\frac{n \theta^{2} b e^{n}}{(1+\theta)}\left(\frac{1+z}{z^{3}}\right) e^{(-\theta / z)}\left(1-\left(1+\frac{\theta}{(1+\theta) z}\right) e^{(-\theta / z)}\right)^{-b-1} e^{-n\left(1-(1+(\theta /(1+\theta) z)) e^{(-\theta / z)}\right)^{-b}}
$$

and 


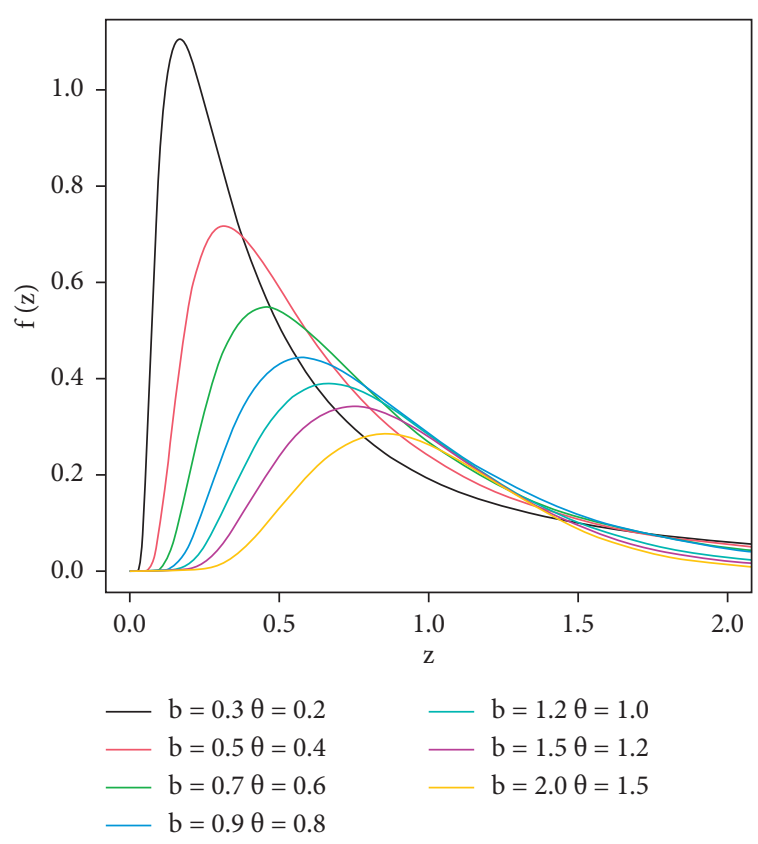

Figure 1: The pdf of the TIIFILi model for various parameter values.

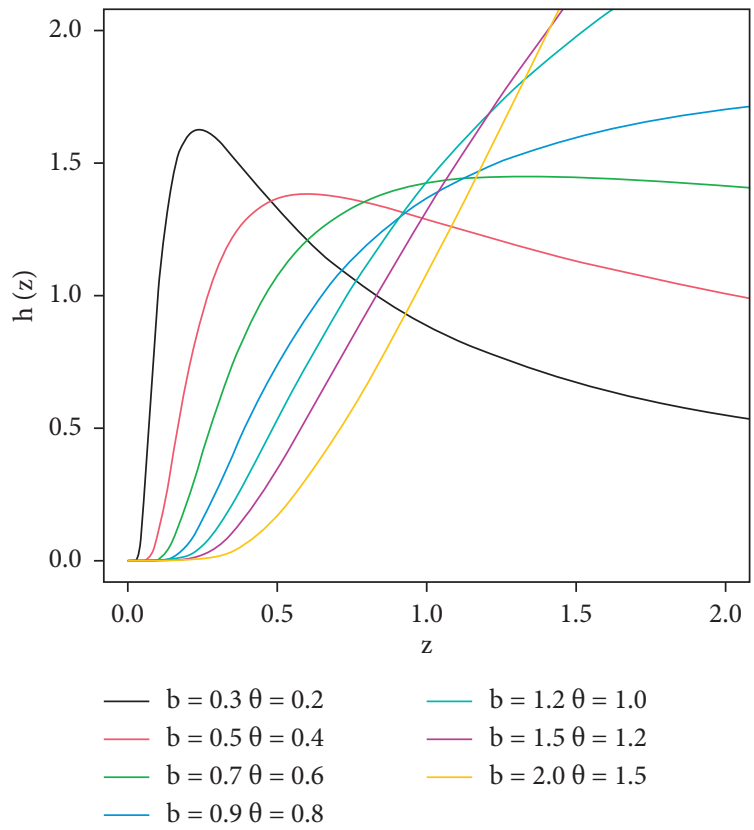

Figure 2: The cdf of the TIIFILi model for various parameter values. 


$$
f\left(z_{n: n}\right)=\frac{n \theta^{2} b e}{(1+\theta)}\left(\frac{1+z}{z^{3}}\right) e^{(-\theta / z)}\left(1-\left(1+\frac{\theta}{(1+\theta) z}\right) e^{(-\theta / z)}\right)^{-b-1} e^{-\left(1-(1+(\theta /(1+\theta) z)) e^{(-\theta / z)}\right)^{-b}}\left(1-e e^{-\left(1-(1+(\theta /(1+\theta) z)) e^{(-\theta / z)}\right)^{-b}}\right)^{n-1} .
$$

The $r^{\text {th }}$ moments of TIITFILi distribution are defined as follows:

$$
\begin{aligned}
E\left(Z^{r}\right) & =\int_{0}^{\infty} z^{r} f(z ; b, \theta) \mathrm{d} z \\
& =\sum_{k=0}^{\infty} \mathbb{C}_{k} \int_{0}^{\infty} z^{r}\left(\frac{1+z}{z^{k+3}}\right) e^{(-\theta(j+1) / z)} \mathrm{d} z \\
& =\sum_{k=0}^{\infty} \mathbb{C}_{k} \int_{0}^{\infty}\left(z^{r-k-3}+z^{r-k-2}\right) e^{(-\theta(j+1) / z)} \mathrm{d} z .
\end{aligned}
$$

$$
\text { Letting } \quad y=(\theta(j+1) / z), \quad z=(\theta(j+1) / y) \text {, }
$$
$d z=\left(-\theta(j+1) / y^{2}\right) d y$, and simplifying further, then

$$
\begin{aligned}
E\left(Z^{r}\right)= & \sum_{k=0}^{\infty} \mathbb{C}_{k} \int_{0}^{\infty}\left(\left(\frac{\theta(j+1)}{y}\right)^{r-k-3}+\left(\frac{\theta(j+1)}{y}\right)^{r-k-2}\right) e^{-y} \frac{\theta(j+1)}{y^{2}} \mathrm{~d} y, \\
E\left(Z^{r}\right)= & \sum_{k=0}^{\infty} \mathbb{C}_{k}(\theta(j+1))^{r-k-2} \int_{0}^{\infty}\left(y^{k-r+1}+\theta(j+1) y^{k-r}\right) e^{-y} d y, \\
& \sum_{k=0}^{\infty} \mathbb{C}_{k}(\theta(j+1))^{r-k-2}(\Gamma(k-r+2)+\theta(j+1) \Gamma(k-r+1)) .
\end{aligned}
$$

The moment generating function of TIITFILi model can be calculated by

$$
M_{Z}(t)=\sum_{r=0}^{\infty} \frac{t^{r}}{r !} E\left(Z^{r}\right)=\sum_{r, k=0}^{\infty} \frac{t^{r}}{r !} \mathbb{C}_{k}(\theta(j+1))^{r-k-2}(\Gamma(k-r+2)+\theta(j+1) \Gamma(k-r+1)), \quad r<k+2 .
$$

The incomplete moments, for example, $\varpi_{s}(t)$ are provided by

$$
\varpi_{s}(t)=\int_{0}^{t} z^{s} f(z ; b, \theta) d z
$$

$$
\varpi_{s}(t)=\sum_{k=0}^{\infty} \mathbb{C}_{k} \int_{0}^{t} z^{s}\left(\frac{1+z}{z^{k+3}}\right) e^{(-\theta(j+1) / z)} \mathrm{d} z
$$

The lower incomplete gamma function is then used to produce

Using (10), then, $\varpi_{s}(t)$ can be taken, the next formula

$$
\varpi_{s}(t)=\sum_{k=0}^{\infty} \mathbb{C}_{k}(\theta(j+1))^{s-k-2}\left(\nu\left(k-s+2, \theta(j+1) t^{-1}\right)+\theta(j+1) \nu\left(k-s+1, \theta(j+1) t^{-1}\right)\right), \quad s<k+2 .
$$


TABle 1: Analysis results for the first data.

\begin{tabular}{|c|c|c|c|c|c|c|}
\hline Model & MLEs (SErs) & $\% 1$ & $\approx 2$ & $\% 3$ & $\approx 4$ & $\% 5$ \\
\hline TIITFILi & $\begin{array}{c}\widehat{b}=0.254(0.0396) \\
\widehat{\theta}=2.625(0.804) \\
\widehat{\alpha}=0.161(0.282)\end{array}$ & 162.44 & 328.88 & 327.835 & 329.325 & 329.777 \\
\hline APTEE & $\begin{array}{c}\widehat{\beta}=2.01 \times 10^{-4}(0.024) \\
\widehat{\gamma}=0.011(0.022)\end{array}$ & 176.631 & 359.262 & 357.694 & 360.186 & 360.607 \\
\hline APTLi & $\begin{array}{c}\widehat{\alpha}=0.1(0.1037) \\
\widehat{\gamma}=0.011(0.024)\end{array}$ & 183.415 & 370.83 & 369.784 & 371.274 & 371.727 \\
\hline PLi & $\begin{array}{c}\hat{\beta}=1.525(0.155) \\
\widehat{\theta}=2.63 \times 10^{-3}\left(2.058 \times 10^{-3}\right)\end{array}$ & 195.999 & 395.999 & 394.953 & 396.443 & 396.895 \\
\hline
\end{tabular}

In this case, $v(s, t)$ is the lower incomplete gamma The Lorenz and Bonferroni curves are given by function.

$$
\begin{aligned}
& L_{F}(z)=\frac{\int_{0}^{t} z f(z ; b, \theta) \mathrm{d} z}{E(Z)}=\frac{\sum_{k=0}^{\infty} \mathbb{C}_{k}(\theta(j+1))^{-k-1}\left(\nu\left(k+1, \theta(j+1) t^{-1}\right)+\theta(j+1) \nu\left(k, \theta(j+1) t^{-1}\right)\right)}{\sum_{k=0}^{\infty} \mathbb{C}_{k}(\theta(j+1))^{-k-1}(\Gamma(k+1)+\theta(j+1) \Gamma(k))}, \\
& B_{F}(z)=\frac{\int_{0}^{t} z f(z ; b, \theta) \mathrm{d} z}{E(z) F(z ; b, \theta)}=\frac{L_{F}(z)}{F(z ; b, \theta)} \\
& =\frac{\sum_{k=0}^{\infty} \mathbb{C}_{k}(\theta(j+1))^{-k-1}\left(\nu\left(k+1, \theta(j+1) t^{-1}\right)+\theta(j+1) v\left(k, \theta(j+1) t^{-1}\right)\right)}{\left(1-e e^{-\left(1-(1+(\theta /(1+\theta) z)) e^{(-\theta / z)}\right)^{-b}}\right)\left(\sum_{k=0}^{\infty} \mathbb{C}_{k}(\theta(j+1))^{-k-1}(\Gamma(k+1)+\theta(j+1) \Gamma(k))\right)} .
\end{aligned}
$$

\section{Maximum Likelihood Estimation}

Assume that $Z_{1}, Z_{2}, \ldots, Z_{n}$ is a random sample of size $n$ from a population with TIITFILi pdf and that the log-likelihood function is provided by

$$
\log L=n+n \log (b)+2 n \log (\theta)-n \log (1+\theta)+\sum_{i=1}^{n} \log \left(\frac{1+z_{i}}{z_{i}^{3}}\right)-\theta \sum_{i=1}^{n} \frac{1}{z_{i}}-(b+1) \sum_{i=1}^{n} \log \left(N_{i}\right)-\sum_{i=1}^{n}\left(N_{i}\right)^{-b}
$$

The score functions which correspond to equating the first-order partial derivative of the last equation to zero is given by

$$
\begin{gathered}
\frac{\partial \log L}{\partial b}=\frac{n}{b}-\sum_{i=1}^{n} \log \left(N_{i}\right)+\sum_{i=1}^{n}\left(N_{i}\right)^{-b} \ln \left(N_{i}\right) \\
\frac{\partial \log L}{\partial \theta}=\frac{2 n}{\theta}-\frac{n}{1+\theta}-\sum_{i=1}^{n} \frac{1}{z_{i}}-(b+1) \sum_{i=1}^{n} \frac{M_{i}}{N_{i}}+b \sum_{i=1}^{n} \frac{M_{i}}{N_{i}^{b+1}},
\end{gathered}
$$

where $N_{i}=1-\left(1+\left(\theta /(1+\theta) z_{i}\right)\right) e^{\left(-\theta / z_{i}\right)}, \quad M_{i}=\left(\partial N_{i} / \partial \theta\right)$ are the solutions, say $\widehat{b}$ and $\widehat{\theta}$. The maximum likelihood estimators of the TIITFILi distribution correspond to the scoring functions. The score functions, on the other hand, are nonlinear functions; the numerical values of the maximum likelihood estimates may be derived using the Newton Raphson iterative optimisation technique.

\section{Modelling to Data Sets}

To describe the performance of the TIITFILi model in reality, actual data sets are explored. The whole first set of data comes from [23]. The outcomes of the fits are compared in data set to the power Li (PLi) by [24], alpha power transformed Li (APTLi) by [25], and APT extended exponential (APTEE) by [26] models.

Statistics measures such as minus log-likelihood ( $\approx 1)$, Akaike information criterion (IC) $(\sigma 2)$, Bayesian IC (ð3), corrected AIC (ð4), and Hannan-Quinn IC (ð5) are obtained. Several criteria are used to compare the TIITFILi model's performance against those of other models.

The maximum likelihood estimates (MLEs), standard errors (SErs) of parameters, and the above statistics measures for the both data sets are given in Table 1. Figures 3-6 provide further information. 


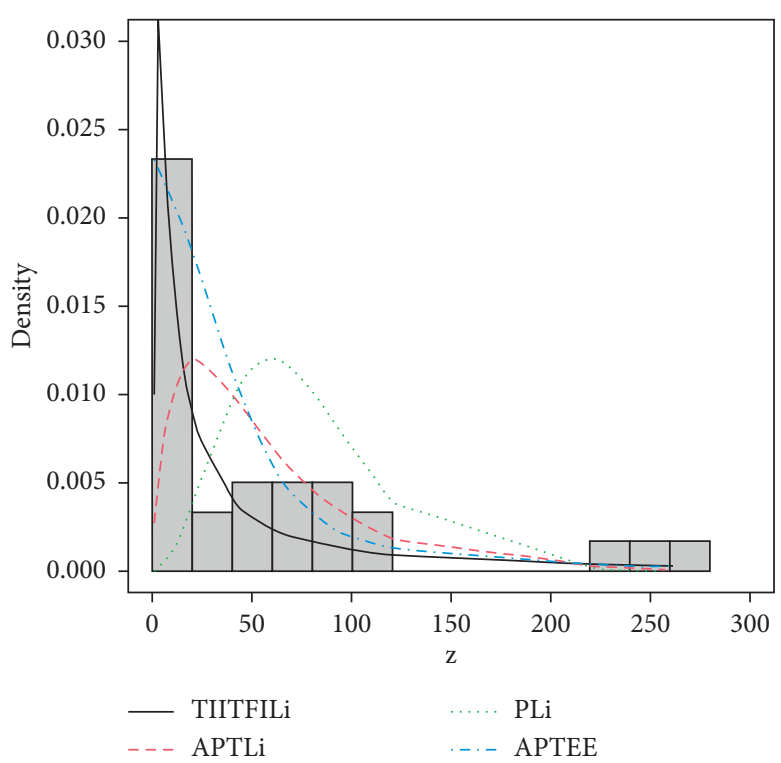

FIgURE 3: Estimated pdf of the TIITFILi and other competing models for first data.

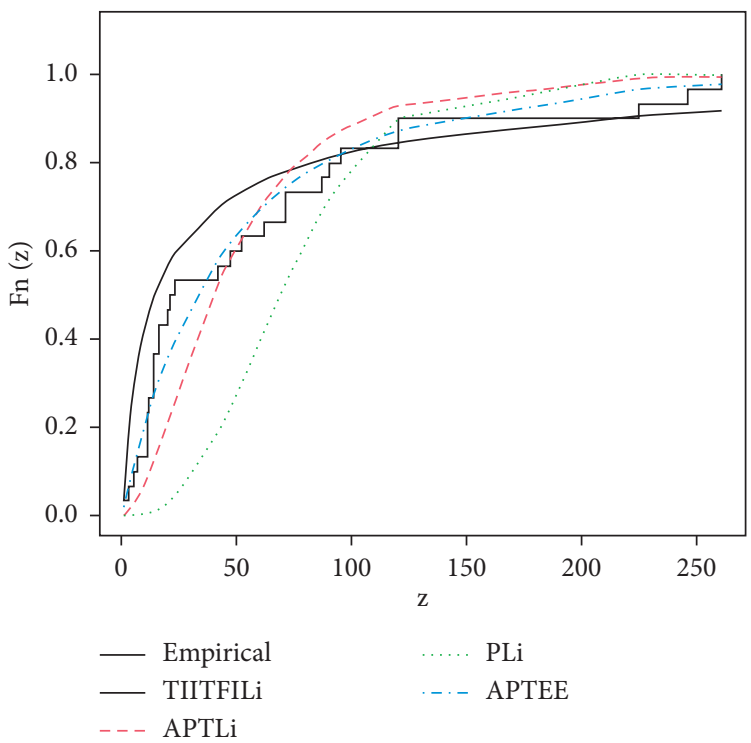

FIgURE 4: Estimated cdf of the TIITFILi and other competing models for the data. 


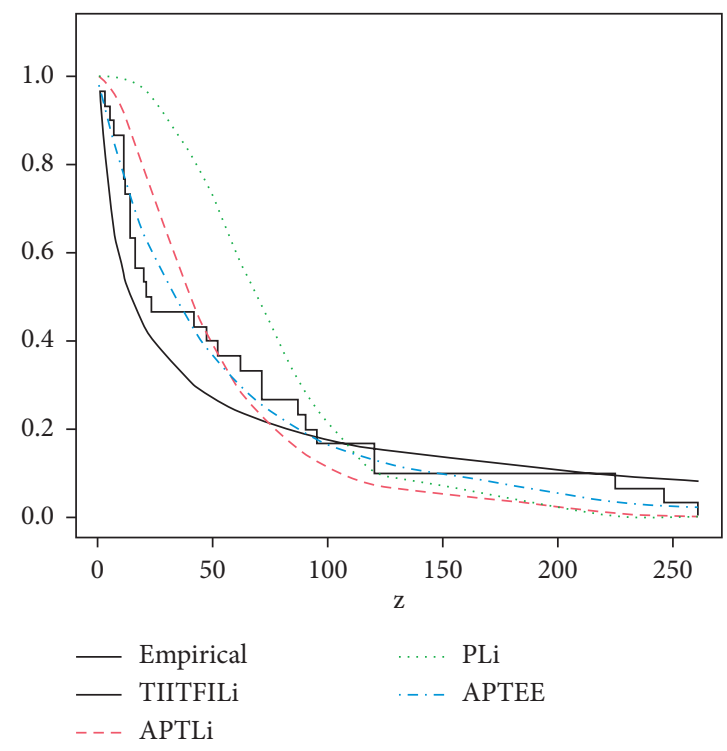

FIGURE 5: Estimated sf of the TIITFILi and other competing models for the data.

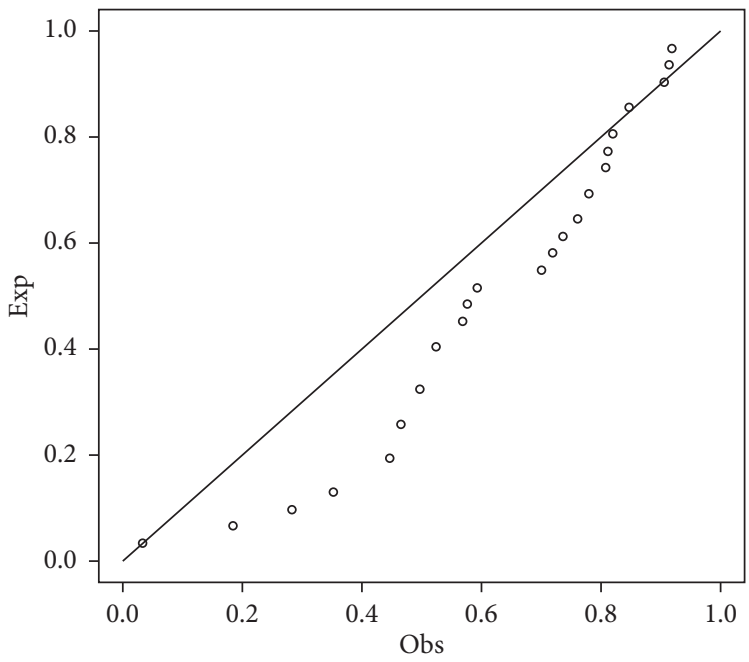

Figure 6: PP plots of the TIITFILi for the data.

Table 1 reveals that the TIITFILi model is best suitable than the APTLi, APTEE, and PLi models. Figures 3 to 6 attempt to estimate pdfs, cdfs, sfs, and pp plots for the fitted models. We infer that now the TIITFILi model fits the data set better.

\section{Conclusion}

This research suggested a novel two-parameter truncated Fréchet inverted Lindley model (TIITFILi) distribution for modelling engineering data and other applications. The TIITFILi model generalizes and extends the inverted Lindley distribution. The TIITFILi distribution's hazard rate might be increasing, unimodal, and J-shaped. Mathematical properties of the new model such as ordinary moments, incomplete moments, and the quantile function, order statistics, are discussed. The maximum likelihood approach is used to estimate the parameters of the new distribution. The novel distribution's value and potential are proven by comparing its fit to a real-world data set to those of existing distributions. According to the goodness-of-fit statistics, the new distribution fits better than the other competing distributions.

\section{Abbreviations}

T: $\quad$ Truncated

TIITFG: Type II truncated Fréchet-G

TPLoG: Truncated power Lomax-G

ILi: $\quad$ Inverted Lindley

APTILi: Alpha power transformed inverted Lindley

hrf: $\quad$ The hazard rate function

cdf: Cumulative distribution function

pdf: Density function 
MLEs: Maximum likelihood estimates

SErs: $\quad$ Standard errors

APTEE: Alpha power transformed extended exponential

PLi: $\quad$ Power Lindley

APTLi: Alpha power transformed Lindley.

\section{Data Availability}

Please contact the relevant author if you would like to acquire the numerical data set used to perform the study presented in the paper.

\section{Conflicts of Interest}

The author declares no conflicts of interest.

\section{References}

[1] S. H. Abid and R. K. Abdulrazak, "[0, 1] truncated Fréchet-G generator of distributions," Applied Mathematics, vol. 7, no. 3, pp. 51-66, 2017.

[2] H. Najarzadegan, M. H. Alamatsaz, and S. Hayati, "Truncated Weibull-G more flexible and more reliable than beta-G distribution," International Journal of Statistics and Probability, vol. 6, no. 5, pp. 1-17, 2017.

[3] M. A. Aldahlan, "Type II Fréchet generated family of distributions," International Journal of Mathematics And its Applications, vol. 7, pp. 221-228, 2019.

[4] R. Bantan, C. Chesneau, F. Jamal, I. Elbatal, and M. Elgarhy, "The truncated Burr X-G family of distributions: properties and applications to actuarial and financial data," Entropy, vol. 23, no. 8, pp. 1-24, 2021.

[5] A. S. Hassan, M. A. Sabry, and A. M. Elsehetry, "A new family of upper-truncated distributions: properties and estimation," Thailand Statistician, vol. 18, no. 2, pp. 196-214, 2020

[6] A. Hassan, M. Sabry, and A. Elsehetry, "A new probability distribution family arising from truncated power Lomax distribution with application to Weibull model," Pakistan Journal of Statistics and Operation Research, vol. 16, no. 4, pp. 661-674, 2020.

[7] A. Alzaatreh, M. A. Aljarrah, M. Smithson et al., "Truncated family of distributions with applications to time and cost to start a business," Methodology and Computing in Applied Probability, vol. 23, no. 1, pp. 5-27, 2021.

[8] M. Akbarinasab, A. R. Arabpour, and A. Mahdavi, "Truncated log-logistic family of distributions," Journal of Biostatistics and Epidemiology, vol. 5, no. 2, pp. 137-147, 2019.

[9] M. Ç. Korkmaz, M. Alizadeh, H. M. Yousof, and N. S. Butt, "The generalized odd Weibull generated family of distributions:statistical properties and applications," Pakistan Journal of Statistics and Operation Research, vol. 14, no. 3, pp. 541-556, 2018.

[10] S. Rezaei, B. B. Sadr, M. Alizadeh, and S. Nadarajah, "ToppLeone generated family of distributions: properties and applications," Communications in Statistics-Theory and Methods, vol. 46, no. 6, pp. 2893-2909, 2017.

[11] M. M. Badr, I. Elbatal, F. Jamal, C. Chesneau, and M. Elgarhy, "The transmuted odd Fréchet-G family of distributions: theory and applications," Mathematics, vol. 8, pp. 1-20, 2020.

[12] M. A. Aldahlan, F. Jamal, C. Chesneau, M. Elgarhy, and I. Elbatal, "The truncated Cauchy power family of distributions with inference and applications," Entropy, vol. 22, pp. 1-25, 2020.
[13] V. K. Sharma, S. K. Singh, U. Singh, and V. Agiwal, "The inverse Lindley distribution: a stress-strength reliability model with application to head and neck cancer data," Journal of Industrial and Production Engineering, vol. 32, no. 3, pp. 162-173, 2015.

[14] S. H. Alkarni, "Extended inverted Lindley distribution: properties and application," Springer-Plus, vol. 4, pp. 1-13, 2015.

[15] V. K. Sharma, S. K. Singh, U. Singh, and F. Merovci, "The generalized inverse Lindley distribution: a new inverse statistical model for the study of upside-down bathtub data," Communications in Statistics-Theory and Methods, vol. 45, no. 19, pp. 5709-5729, 2016.

[16] K. V. P. Barco, J. Mazucheli, and V. Janeiro, "The inverse power Lindley distribution," Communications in Statistics-Simulation and Computation, vol. 46, no. 8, pp. 6308-6323, 2017.

[17] V. K. Sharma and P. Khandelwal, "On the extension of inverted Lindley distribution," Journal of Data Science, vol. 15, pp. 205-220, 2017.

[18] J. Kantalo and J. Suntornchost, "A two-parameter weighted inverted Lindley distribution and applications," Journal of Applied Statistics and Information Technology, vol. 2, no. 2, pp. 1-14, 2017.

[19] S. Dey, M. Nassar, and D. Kumar, “Alpha power transformed inverse Lindley distribution: a distribution with an upsidedown bathtub-shaped hazard function," Journal of Computational and Applied Mathematics, vol. 348, pp. 130-145, 2019.

[20] M. Eltehiwy, "Extended exponentiated inverse Lindley distribution: model, properties and applications," Journal of the Indian Society for Probability and Statistics, vol. 20, no. 2, pp. 281-300, 2019.

[21] M. Eltehiwy, "Logarithmic inverse Lindley distribution: model, properties and applications," Journal of King Saud University Science, vol. 32, no. 1, pp. 136-144, 2020.

[22] R. Bantan, A. S. Hassan, and M. Elsehetry, "Generalized Marshall Olkin inverse Lindley distribution with applications," Computers, Materials \& Continua, vol. 64, no. 3, pp. 1505-1526, 2020.

[23] H. Linhart and W. Zucchini, Model Selection, Wiley, New York, NY, USA, 1986.

[24] M. E. Ghitany, D. K. Al-Mutairi, N. Balakrishnan, and L. J. AlEnezi, "Power Lindley distribution and associated inference," Computational Statistics \& Data Analysis, vol. 64, pp. 20-33, 2013.

[25] S. Dey, I. Ghosh, and D. Kumar, "Alpha power transformed Lindley distribution: properties and associated inference with application to earthquake data," Annals of Data Science, vol. 6, pp. 1-28, 2018.

[26] A. S. Hassan, R. E. Mohamd, M. Elgarhy, and A. Fayomi, "Alpha power transformed extended exponential distribution: properties and applications," The Journal of Nonlinear Science and Applications, vol. 12, pp. 239-251, 2019. 